\title{
On Image Processing Algorithm for Registration of Three-Dimensional Gas Bubble Movement Using a High Speed Camera
}

\author{
Jakub AUGUSTYNIAK ${ }^{1}$, Dariusz M. PERKOWSKI ${ }^{2}$
}

\author{
Faculty of Mechanical Engineering, Department of Mechanics and Applied Computer Science, Bialystok \\ University of Technology \\ ul. Wiejska Street 45C, 15-351 Bialystok, Poland
}

\begin{abstract}
:
The paper deals with an imaging computer tomography method based on simple image processing techniques for two phase flow analysis. Moreover, it has been presented the algorithm of 3D bubble trajectory reconstruction using a single high speed camera and the system of mirrors. In the experiment a glass tank filled with distilled water was used. The nozzle through which the bubbles were generated was placed in the center of the tank bottom. Through the use of basic image processing and analysis techniques such as noise reduction, smoothing, edge detection and few algorithms like close contour filling, tracking single bubble etc. proposed in paper it became possible to draw out 3D trajectories for gas bubble paths in liquid. In the paper the measurement error of imaging computer tomography method has been estimated. The maximum measurement error recorded for this method was within the limits $\pm 0,65$ [mm] for a certain set of parameters like: resolution, mirror angle and deviation error of $\mathrm{z}$ axis from the $90^{\circ}$ vertical line. Trajectories of subsequently departing bubbles were visualized in the form of figures.
\end{abstract}

Key words: Image processing, OpenCV, triangulation, Kalman filter, gas bubble, nozzle

\section{Introduction}

In the paper Shew and Poncet (Shew et al., 2006) presented that the path of bubbles changes due to the increase of bubbles diameter and thereby its shape. Many scientists studied the behavior of bubbles rising in water, however the most common problem that occurred was the proper finding and tracking of individual gas bubbles (Duineveld et al., 1995; de Vries et al., 2002; Aybers et al., 1969; Hassan et al., 2008; Ellingsen et al., 2001). Authors (Dzienis et al.,2014) show that the air pressure, liquid movement inside the nozzle and the volume of the plenum chamber have a significant impact on the bubble trajectory. Bubbles that depart aperiodic show a significant deviation of bubble trajectory from a straight line (Dzienis et al., 2012). In article (Augustyniak et al., 2015) the authors discovered that the predictability of bubble path projections varies depending on the plane which has been chosen for the projection. Scientist have been struggling to optimize one process allowing to fully visualize the path of departing bubbles. Many methods for virtual reconstruction of the bubbles movement have been presented in the past years (Rostamzadeh et al., 2016, Wacławczyk et al., 2002). The complexity of different algorithms like two-dimensional decimated wavelet transform, denoising based on wavelet transform or even decomposition into angular and translational components (see, for example Ding et al., 2007; Lin et al., 2007; Wada et al., 2006; Lunde et al., 1997; Tian et al., 2013; Favaro et al., 2000). In this paper an image processing method has been presented using a high speed camera and mirror system which it was applied to determine of 3D trajectory of bubbles movement.

Under the concept of image processing and analysis we can find many methods highlighting the features necessary from the observer point of view. It is an essential element of working with digital images. They provide the basis for subsequent operations like: color or shape detection, tracking or recognition. Image processing and analysis is a process of extraction of information important to the observer. To achieve this a computer vision library was used, the OpenCV. It provides a common infrastructure for computer vision applications and accelerates the use of machine perception in the commercial products 
(opencv.org). The OpenCV library is equipped with more than 2500 algorithms allowing to identify objects, track motion, segmentation, recognition or even provides a human-computer interaction (HCI) (Kaehler et al., 2008).

The main aim of the present paper was to create an efficient and reliable imaging computer tomography software for determining the 3D gas bubble trajectory in liquid.

Based on the paper (Ohl et al., 2003) an experimental setup was made which consisted of a single high speed camera and a set of mirrors. Recorded images have been processed using algorithms available in the OpenCV library. The presented algorithm allows to identify individual bubbles reducing the evaluation time and consumption of computer memory. To predict the position of the interest zone the Kalman filter was applied. The triangulation method was used for calculation of 3D bubble coordinates. The measurement error has been estimated.

\section{Experimental Setup And Data Analysis}

Fig. 1 illustrates the schema of the experimental setup (glass tank ( $300 \times 150 \times 500 \mathrm{~mm})$ filled with distilled water). A brass nozzle with an internal diameter of $1.1 \mathrm{~mm}$ and $70 \mathrm{~mm}$ in length was placed in the center of the tank bottom. The air was provided from a flow meter connected to a compressed air system. The video was recorded with a high speed camera Phantom v1610 with $600 \mathrm{fps}$ placed in front of a right-angled mirror (Fig.1). To obtain a clear image the glass tank was illuminated from both sides by LED panels. "Milk" glass has been used to disperse the light and to get a uniform background. A three mirror system was used which gave the possibility to obtain two different projections of the same scene on one single frame recorded with the camera. For this purpose, two flat mirrors were placed on the sides of the glass tank. The reflected projections were focused by the rectangular third mirror.

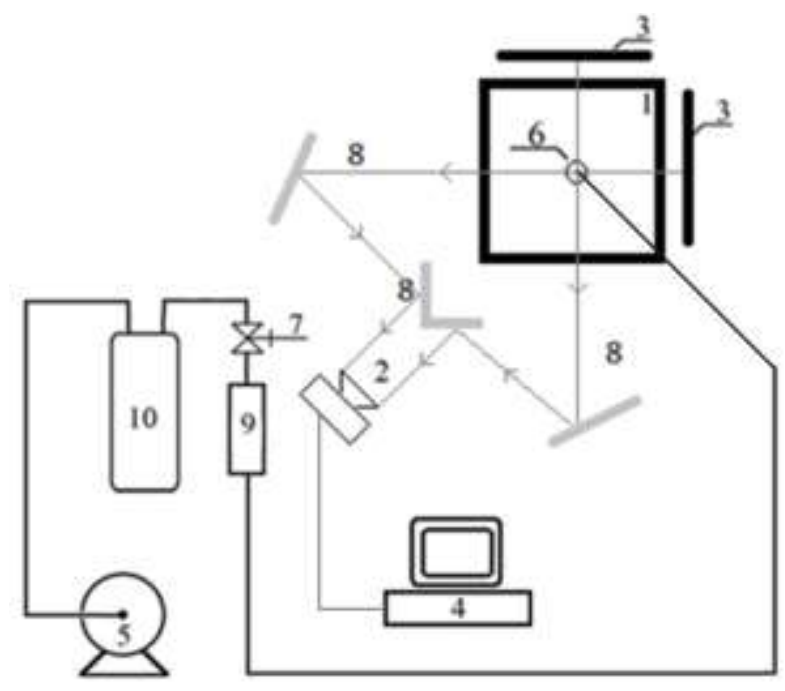

Fig. 1. Scheme of the experimental setup: 1 - glass tank, 2 - camera, 3 - light panel, 4 - data acquisition station, 5 - air pump, 6 - nozzle, 7 - air valve, 8 - mirrors, 9 - flow meter, 10 - air tank

The main problem for a proper identification of three-dimensional gas bubble movement was the designation of center of each bubble from a set of contours describing a single bubble. In order to subtract information from images basic image processing techniques are needed like: image filtration, edge detection, contour finding.

\subsection{Image filtration}

Image filtration is one of the basic functions of image processing aimed at the removal of undesirable elements such as noise or distortion. It can be used also to bring out features that play an important role in the further work. Noise in images is usually caused by environmental conditions like light or temperature. In the experiment the main source of noise was the light (its glow, reflection). In order to obtain a noise free 
image with minimum changes in the original images value a statistic filter should be considered. To get rid of the noise a median filter was used. The filter selects the average pixel value (median) checking each pixel in the image and compares it with its surroundings. The result is the removal of pixels deviating from the ambient (Fig. 2).

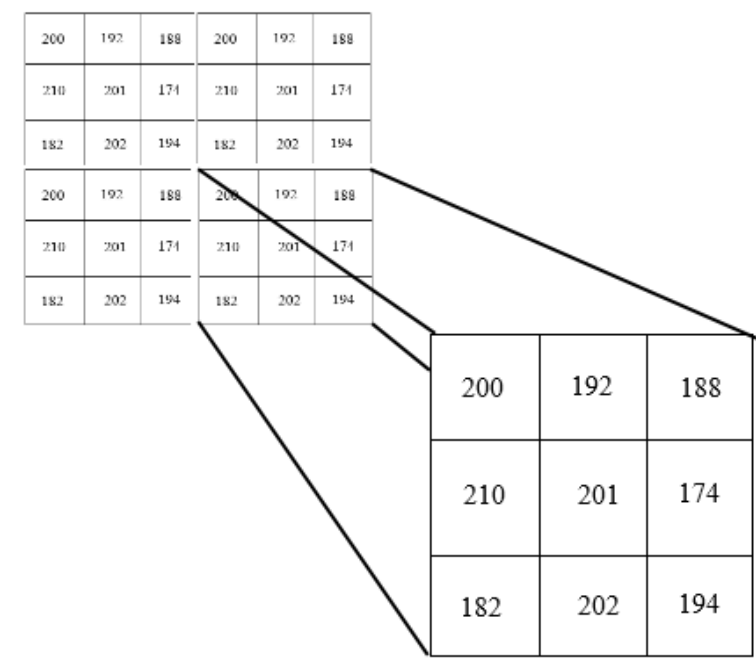

\author{
Neighbourhood pixel \\ values: \\ $174,182,188,192,194$, \\ $200,201,202,210$ \\ Median value: 194 \\ Pixel value $201 \rightarrow 194$
}

Fig. 2. Principle of median filter (Kaehler et al., 2008)

With the help of the median filter it was possible to overcome the presence of micro bubbles during the departure of main bubbles from the nozzle. Without the filter every small inaccuracy occurring in the experiment would make it much harder to correctly determine the path of bubbles.

\title{
2.2. Edge detection
}

Edge detection is a very significant step in image processing. Edges carry important information about the objects in the image. In fact, the edges perceive as a smooth, regular surfaces with different reflectance properties. Authors (Wróbel et al., 2004) defined edges as a line separating regions of different brightness's, different levels of saturation. Therefor it is the transition region between two levels of brightness's.

In the experiment a multi-stage edge detecting algorithm has been used, the Canny edge filter. The edge finding process started from the convolution between the non-noise image with two $3 \times 3$ matrixes in order to determine the intensity gradient of the image. The used matrixes were (Canny 1986; Ding et al., 2001):

$$
\begin{gathered}
G_{x}=\left[\begin{array}{ccc}
-1 & 0 & 1 \\
-2 & 0 & 2 \\
-1 & 0 & 1
\end{array}\right], G_{y}=\left[\begin{array}{ccc}
-1 & -2 & -1 \\
0 & 0 & 0 \\
1 & 2 & 1
\end{array}\right], \\
G=\sqrt{G_{x}^{2}+G_{y}^{2}}, \theta=\arctan \left(\frac{G_{y}}{G_{x}}\right) .
\end{gathered}
$$

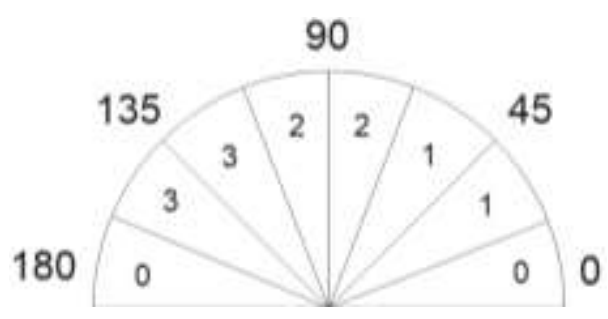

Fig. 3. Canny edge filter gradient orientation (Canny 1986) 
The gradient orientation is rounded to one of four possible angles (namely $0,45,90$ or 135). Next two thresholds (upper and lower) are setup to reduce number of false edges. When the pixel's brightness is below the lower threshold, the pixel is not considered. When the brightness is higher than the upper threshold, the pixel is treated as belonging to the edge. When the pixel brightness is between the upper and the lower threshold, the pixel is treated as belonging to the edge when at least one of its neighbours belongs to the edge. The proper selection of the thresholds value was crucial upon receiving a clear bubble edge image. Authors tested also other common edge detection filters like the Sobel or Prewitt filters, however the results did not come as expected (algorithm could not find properly closed outer contours of the gas bubbles - reasons were the origins of the filters, one works better horizontally the other vertically) therefore the Canny edge filter was chosen.

\subsection{Full contour finding}

In order to trace the movement of bubbles an edge detection filter was used. The obtained image contained many lines presenting edges. For a proper designation of bubbles outer surface, a method for finding the biggest contour was introduced. Each line representing an edge affiliated with the bubble was defined as a contour area. When the contour was not fully closed the area was set to 0. From a set of full contour areas, the biggest one was taken as the outer boundary of the bubbles image.

The reconstruction of the bubble trajectory required the finding of the center of area of a bubble images in each frame. The position of the center of bubble area is presented in Fig. 5.
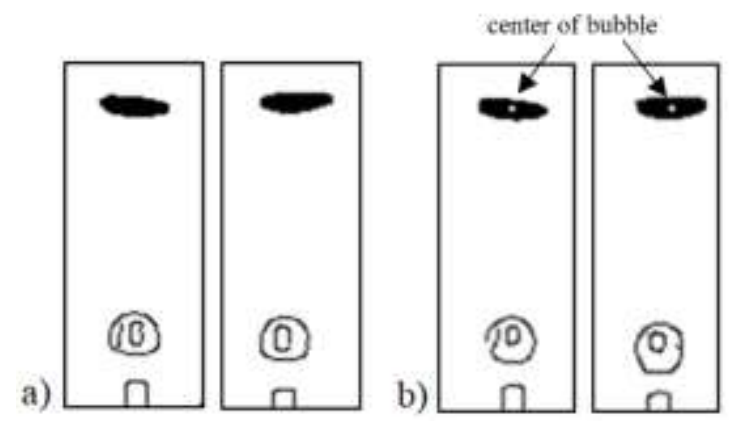

Fig. 5. Position of the center of area of a bubble image in each frame

For a proper finding of center of area of a bubble image the outer contour had to be filled with white pixels. Therefore, an iteration algorithm has been proposed. The algorithm started from finding the first pixel being white (in a binary image pixel with a 255 value). A mark was set in order to signal the beginning of filling procedure. The filling has stopped whenever a second white pixel was found. The procedure was conducted in each $\mathrm{x}$ and $\mathrm{y}$ axis pixel per pixel. Thus this algorithm it was able to get contours picturing bubble images with an even white surface and the position of center of area of the bubble.

\subsection{Identification of individual bubble images}

Previous steps enabled to receive a binary image made of white contours picturing bubble surfaces and the position of center of area. However, this procedure consumes a lot of the computer memory. To reduce the complexity of the method an ROI (Region of Interest) was implemented. The main aim of ROI was to decrease the area in which the whole edge-full contour finding and filling algorithm took place. Through the use of ROI, it was able to identify many subsequently departing bubbles. ROI gave the possibility to measure each bubbles trajectory independently while maintaining order of their movement. In Fig. 6 the identifications process of individual bubble images with help of ROI has been presented. 


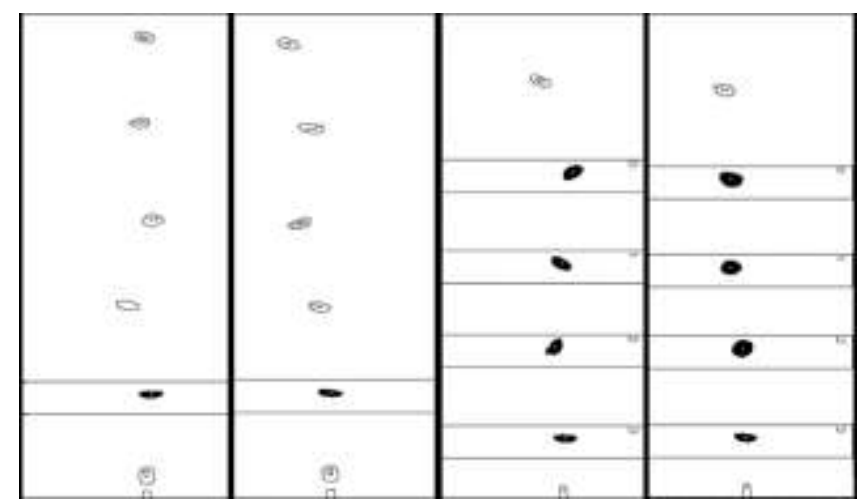

Fig. 6. Identification of individual bubble images with ROI (inverse color)

\subsection{Object tracking (Kalman filter)}

In order to track an object in a video one must find the motion path of an object over time, or simpler in every frame of the video. There are many different technics which are used for tracking objects. A general division of those technics puts them into one of three groups: point tracking, kernel tracking and silhouette tracking. Through the changes that bubbles undertake, the velocity of their movement changes in different stages of the motion. There are three main paths that occur: rectilinear path which appears when the bubble diameter $<0.81[\mathrm{~mm}]$, the bubble zigzag motion is observed when bubble diameter is $0.81-0.88[\mathrm{~mm}]$ and a bubble spiral motion appears for bubble diameter greater than 1.10 [mm] (Kalman 1960). In chapter 2.4 explained that in order to identify each and every bubble an ROI had to be used. In order to keep up with the bubble image displacement and predict the bubbles next position the Kalman filter was used.

The Kalman filter is a linear quadratic estimation algorithm which uses series of measurement observed over time and estimates variables of the underlying system state (Kalman 1960). Kalman filter is a two-step process: prediction and estimation. In the first, the filter creates estimates of the current state of measured variables, then once the next measurement is observed the estimates are updated with the help of a weighted average.

The Kalman filter was set up with 4 dynamic parameters and 2 measurement parameters which were not controlled by the user. The measurement parameters were: $x$ and $y$ location of the bubble. As for the dynamic parameters: $x, y$ location and respectively $V x, V y$ as velocity. The kinematic equations for this situations were (Kalman 1960):

$$
\begin{gathered}
x_{n}=x_{n-1}+V_{x n-1} \Delta t \\
y_{n}=y_{n-1}+V_{y n-1} \Delta t, \\
V x_{n}=V_{x n-1}, \\
V y_{n}=V_{y n-1} .
\end{gathered}
$$

This gave a simple transition matrix Tr:

$$
\operatorname{Tr}=\left[\begin{array}{llll}
1 & 0 & 1 & 0 \\
0 & 1 & 0 & 1 \\
0 & 0 & 1 & 0 \\
0 & 0 & 0 & 1
\end{array}\right]
$$

On this basis it was possible to track bubble images in ROI without losing them. In Fig. 7 the real measured and predicted Kalman position of a gas bubble has been shown. 


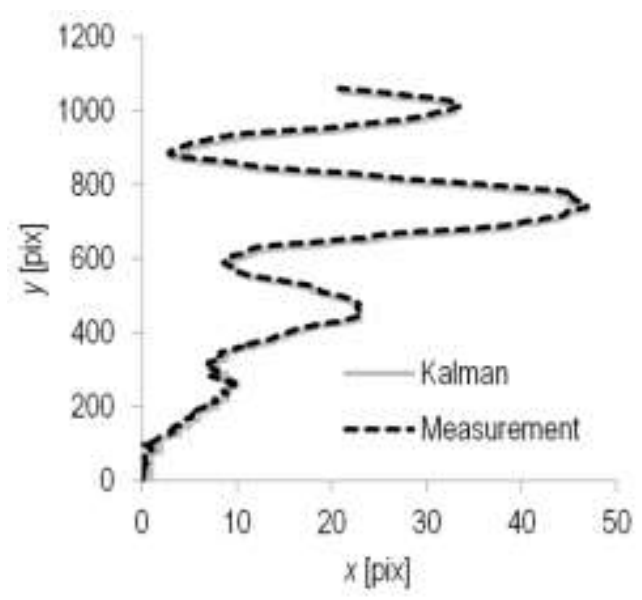

Fig. 7. The result of Kalman filter with real measurement values of bubble trajectory.

As a final result we obtained two separate projections of bubble outer contour and the trajectory of each bubble center of mass in both planes that is in the $x z$ and $y z$. The graphical interpretation of the working to this point algorithm is presented in Fig. 8.
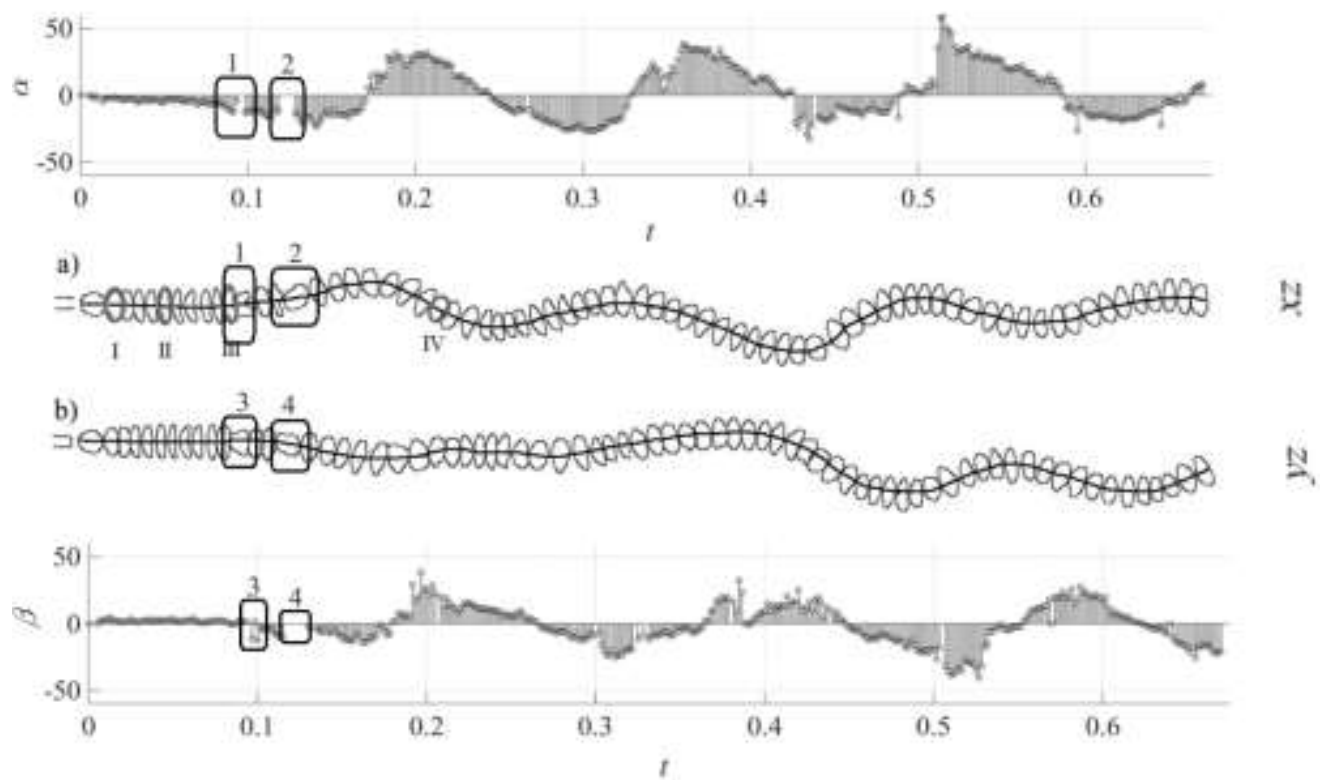

Fig. 8. The result of image processing steps providing a two plane projection (planes $x z$ and $y z$ ) of bubble outer contour representation and the corresponding trajectory of bubble center of mass (additionally added ellipses created from the algorithm from I to IV and the evolution of inclination of bubble deformation).

Having the ability to reconstruct the gas bubble surface and therefore their center of gravity, it is also possible to obtain the major and minor semi-axes of an ellipse drawn onto the contour of the bubble. Additionally it becomes possible to extract the rotation angel of the ellipse. This information can be very useful when building a mathematical model of the process. On Fig. 8 there are four ellipses (marked with I, II, III and IV) drawn onto the raw outer contour of the gas bubble. In Fig. 9 the authors present a better understanding of the ellipse fitting process. 


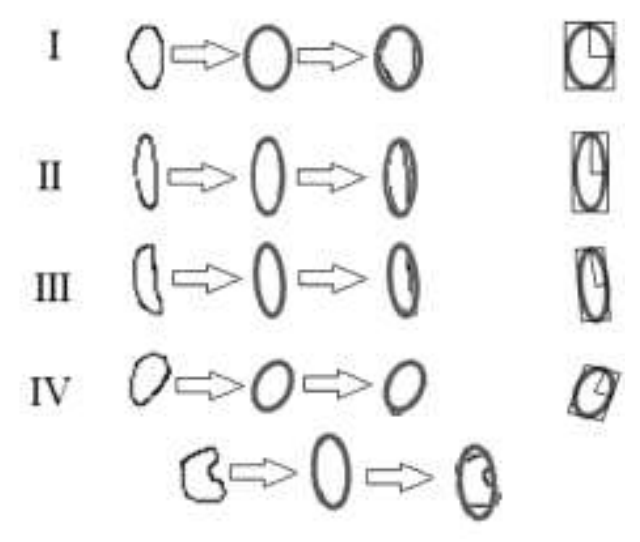

Fig. 9. Bubble contours and the corresponding ellipse with its major and minor semi-axis.

However there is also a risk. Fitting ellipses won't go always as planned. The reason for that are the major distortions that the bubbles surface undertake during the freely rise. An example is pictured in Fig. 9 below the main four examples. What more analyzing the image presenting the tilts of bubbles, one can notice small gaps in the diagram, proving that there are deformations of the bubble which are too complex and too quick in progress in order to fully be reconstructed using ellipse fitting. However such diagrams can be very helpful in building a model or draw from it information about the forces acting on the bubble. The accuracy of determining the shape of a freely rising gas bubble is of great importance in fluid mechanics when creating a mathematical model (see ) and designating some dimensionless numbers for example: Reynolds, Weber, Bond etc. The next major step was to create a three dimensional representation of each individual bubbles trajectory.

\section{Triangulation Method}

The bubble coordinates in the Cartesian coordinates system, which origin is located in the nozzle outlet (see Fig. 8) have been calculated according the following formulas:

$$
\begin{gathered}
X_{c}=\frac{\sin (\beta) x}{\cos (\beta) \sin (\alpha)}, \\
Y_{c}=\frac{\sin (\beta) y}{\cos (\beta) \sin (\alpha)}, \\
Z_{c}=R_{k}-\frac{P_{p 1}+P_{p_{2}}}{2}-\frac{S_{p_{1}}+S_{p 2}}{2} .
\end{gathered}
$$

where: $X c, Y c, Z c$ - are the coordinates in the coordinate system whose origin is located at the nozzle outlet; $R k$ - height of the image; $P p 1, P p 2$ - location of ROI frames; $S p 1, S p 2$ - center of mass of a bubble.

Figure 10 presents the visual interpretation of triangulation method for $Y c$ with marked lines representing ray reflections. The $X c$ coordinate is calculated analytically. 


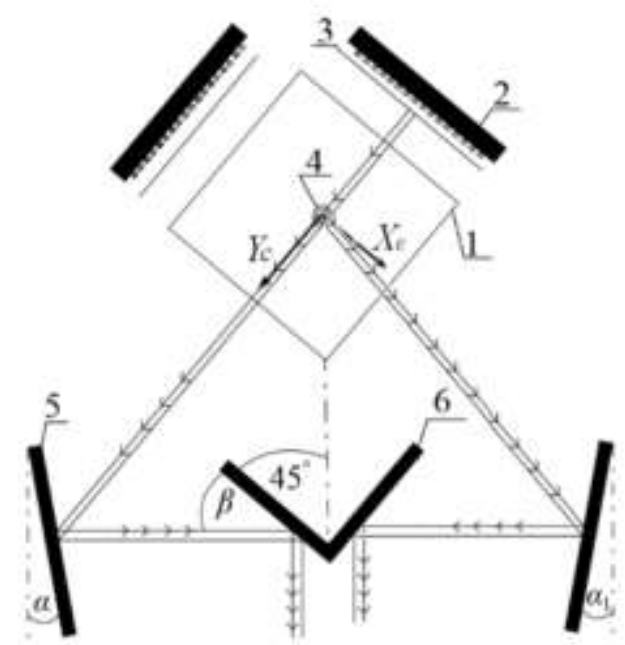

Fig. 10. Visual interpretation of triangulation transformation for a proper $Y c$ and $X c$ (1-glass tank, $2-$ LED panel, 3 - „milk” glass, 4 - nozzle, 5,6 - mirrors)

\section{Measurement Error}

Measurement of position of the center of area was an indirect measurement. To determine the accuracy of the measurement method, the total differential method was used (Jakubiec et al., 2004). The estimation of the errors $\Delta X c$ and $\Delta Y c$ has been made using the following formulas:

$$
\begin{gathered}
\Delta X_{c}=\frac{\partial}{\partial \alpha} X_{c}(\alpha, \beta, x) \Delta \alpha+\frac{\partial}{\partial \beta} X_{c}(\alpha, \beta, x) \Delta \beta+\frac{\partial}{\partial x} X_{c}(\alpha, \beta, x) \Delta x, \\
\Delta Y_{c}=\frac{\partial}{\partial \alpha} Y_{c}(\alpha, \beta, y) \Delta \alpha+\frac{\partial}{\partial \beta} Y_{c}(\alpha, \beta, y) \Delta \beta+\frac{\partial}{\partial y} Y_{c}(\alpha, \beta, y) \Delta y, \\
\Delta Z_{c}=\frac{\partial}{\partial \delta} Z_{c}(\delta, z) \Delta \delta+\frac{\partial}{\partial z} Z_{c}(\delta, z) \Delta z .
\end{gathered}
$$

where: $\Delta \alpha$ and $\Delta \beta$ are the protractors measurement errors which are equal to 5'. The $\Delta x$ and $\Delta y$ were estimated based on the comparison between experimental measurement and the known diameter of a metal ball. The $\Delta \delta$ is the deviation error of $z$ axis from the $90^{\circ}$ vertical line.

The maximum error of $\Delta z$ (in 100 measurements) was less than $\pm 0,5$ [mm]. The maximum measurement error defined by the Eq. (11) - (13) was equal to $\pm 0,65$ [mm]. These errors significantly depend on the image resolution. In the experiment the image resolution was 1280x800 [pix].

Due to the numerous refractions of the light and thus reflected image which then hit the sensor of the camera a calibration method had to use in order to determine the distortion in the system. The main purpose of the calibration in to determine the parameters defining the relationship between the basin system and the system associated with the perspective transformation and parameters related to the camera and optical system. For this reason, a known calibration standard was used, namely the "black white chessboard" presented in Fig. 11. 


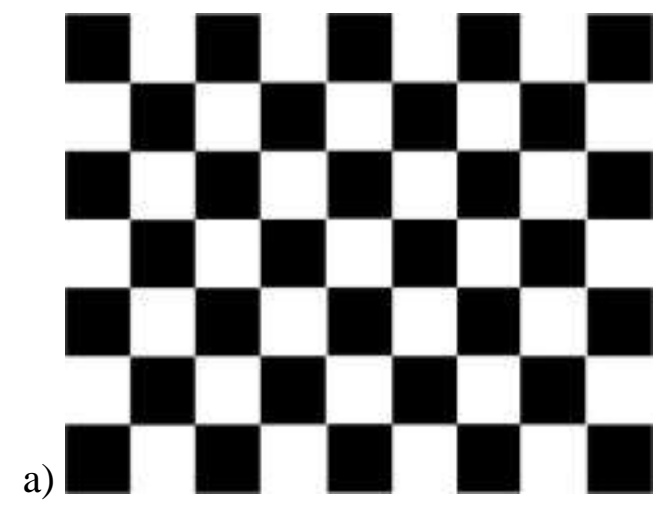

Fig. 1. Calibration standard "chessboard" and the concept of it (1,2 positions of the board).

The numerical values obtained with using the algorithm described above allowed to plot the 3D gas bubble trajectories, see Fig. 12.

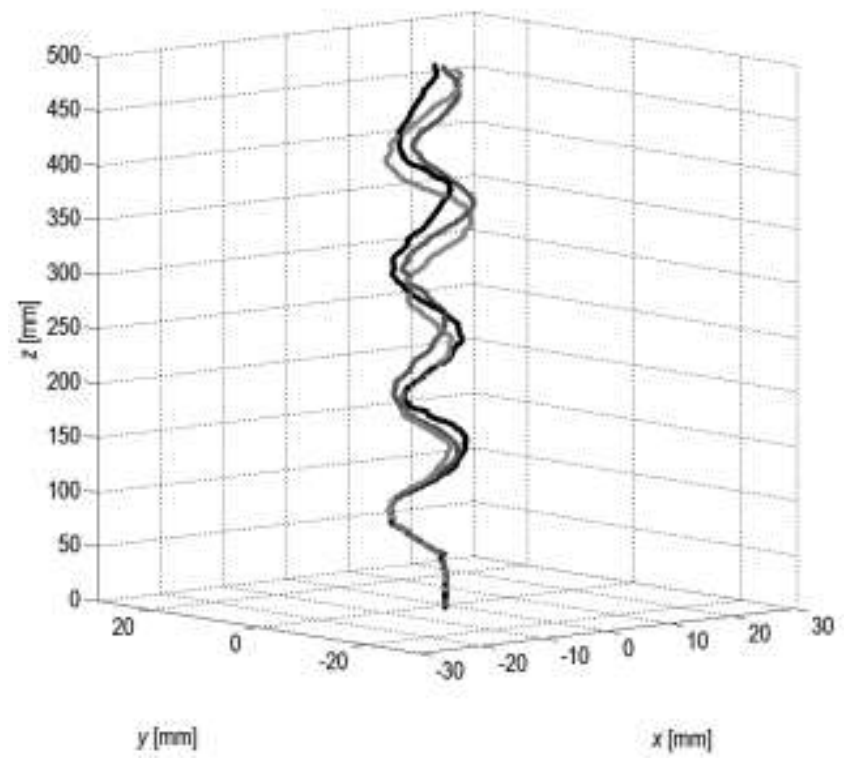

Fig. 12. Three dimensional path reconstruction of three consecutive gas bubbles

\section{Conclusion}

In this paper an imaging computer tomography algorithm for determining the 3D gas bubble trajectory in liquid has been presented. The recordings were taken by a high speed camera the Phantom v1610. The videos had to be analyzed by use of a computer program allowing for a $3 \mathrm{D}$ visualization of the results. The main parts of the program were: reducing noise, proper edge detection, finding full contours and filling them with white pixels, calculation of center of area and tracking. Many factors influenced the efficiency on the software. An important element was the illumination of the tank. The light could not be too bright causing the bubbles to become more vitreous nor emitted at a low-frequency (light pulsation in the recordings). Another thing was the proper threshold value selection. This choice marked the accuracy of every step occurring after the Canny edge filter application. Determining the coordinates of center of area of bubble images properly needed a fully filled bubble contour. To achieve that the iteration process was conducted firstly in the vertical orientation than in the horizontal one. Using the triangulation techniques, the 2D bubble coordinates were transformed into 3D coordinates system. The movies were recorded at $600 \mathrm{fps}$ with a 1280x800 [pix] resolution. In this experiment the maximum measurement error calculated form the (11) (13) equations was within the limits $\pm 0,65[\mathrm{~mm}]$. The most significant contribution in measurement error has the $\Delta x$ and $\Delta y$ measurements. Their values significantly depend on the image resolution. The video recorded with a higher resolution has a smaller error. What is more the presented algorithm can be adapted 
to a system consisting of two nozzles (or more) by transforming the Kalman filter in to directions and reducing the size of ROI. The obtained results in form of three dimensional gas bubble trajectories can be used for some nonlinear analysis methods like multifractal analysis or estimation of Hurst parameter, they can be very helpful in building a mathematical model or in a simulation providing with basic information about the forces and behaviors that the bubble is undergoing.

\section{References}

[1] A.W.G. de Vries, A. Biesheuvel, L van Wijngaarde, 2002, Notes on the path and wake of a gas bubble rising in pure water, International Journal of Multiphase Flow, vol. 28, 11, 1823-1835.

[2] Augustyniak J., Perkowski D.M, Mosdorf R., 2015, Measurement of properties of chaotic bubble paths, International Journal of Heat and Mass Transfer, 85, 732-739.

[3] Aybers N.M., Tapucu A., 1969, The motion of gas bubbles rising through stagnant liquid, Heat and Mass Transfer, vol. 2, 118-128.

[4] C. Lin, M. Gilberston, A. Harrison, 2007, Bubble distribution and behavior in bubbling fluidized beds, Chemical Engineering Science, vol 62, 56-69.

[5] Canny J., 1986, A Computational Approach To Edge Detection, IEEE Trans. Pattern Analysis and Machine Intelligence, 8(6):679-698.

[6] Ding H., Song ZY., 2007, Hilbert-Huang transform based signal analysis for the characterization of gas-liquid two-phase flow, Flow Measurement and Instrumentation, vol. 18, 37-46.

[7] Ding L., Goshtasby A., 2001, On the Canny edge detector, Pattern Recognition, nr. 34, 721-725.

[8] Dzienis P., Mosdorf R., 2014, Stability of periodic bubble departures at a low frequency, Chemical Engineering Science, 109, 171-182.

[9] Dzienis P., Mosdorf R., Wyszkowski T., 2012, The dynamics of liquid movement inside the nozzle during the bubble departures for low air volume flow rate, acta mechanica et automatica, vol.6 no.3.

[10] Ellingsen K., Risso F., 2001, On rise of an ellipsoidal bubble in water: oscillatory paths and liquid induced velocity, Journal of Fluid Mechanics, vol. 440.

[11] Favaro P., Soatto S., 2000, Shape and Radiance Estimation from the Information Divergence of Blurred Images, Lecture Notes in Computer Science, 1842, 755-768.

[12] Hassan N.M.S., Khan M.M.K., Rasul M.G., 2008, A Study of Bubble Trajectory and Drag Coefficient in Water and Non-Newtonian Fluids, Wseas transition on fluid mechanics, vol.3, no.3.

[13] http://opencv.org/about.html.

[14] Jakubiec W., Malinowski J., 2004, Metrologia wielkości geometrycznych, Wydawnictwa NaukowoTechniczne - Wydanie czwarte zmienione, 52-93.

[15] K. Sakakibara, M. Yamada, Y. Miyamoto, 2007, Measurement of the surrounding liquid motion of a single rising bubble using a dual-camera PIV system, Flow Measurement and Instrumentation, vol. $18,211-215$.

[16] Kaehler A., Bradski G., 2008, Learning OpenCV. Computer Vision in C++ with the OpenCV Library. O'Reilly Media.

[17] Kalman, R. E.,1960, A New Approach to Linear Filtering and Prediction Problems, Journal of Basic Engineering, 82 (1): 35-45.

[18] Lunde K., Perkins R.,1997, A new experimental method to analyse the dynamics and the morphology of bubbles in liquids: Application to single ellipsoidal bubbles, Fluids Engineering Division Summer Conference, 3530.

[19] Ohl C.D., Taijink A., Prosperetti A., 2003, The added mass of an expanding bubble, Journal of Fluid Mechanics,, vol. 482, 271-290.

[20] P.C Duineveld, 1995, The rise velocity and shape of bubbles in pure water at high Reynolds number, Journal of Fluid Mechanics, vol. 292, 325-332.

[21] Rostamzadeh A., Jafarpur K., Rad E. G., 2016, Numerical investigation of pool nucleate boiling in nanofluid with lattice boltzmann method, Journal of Theoretical and Applied Mechanics, 54, 3, pp. 811-825

[22] S. Wada, H. Kikura, M. Aritomi, 2006, Pattern recognition techniques in multiphase flow, Flow Measurement and Instrumentation, vol. 17, 207-224. 
[23] Shew W.L., Poncet S., Pinton J-F., 2006, Force measurements on rising bubbles, Journal of Fluid Mechanics, vol. 569, 51-60.

[24] Taubin G., Moreno D., Build Your Own Desktop 3D Scanner (Chap. 2 \& 4).

[25] Tian Y., Zheng Ch., Ke Q., 2013, A Bubble Detection Algorithm based on Sparse and Redundant Image Processing, Telecommunication Computing Electronics and Control, Vol. 11, No. 6, pp. 2983 $-2990$.

[26] Wacławczyk M., Pozorski J., 2002, Two-point velocity statistics and the POD analysis of the nearwall region in a turbulent channel flow, Journal of Theoretical and Applied Mechanics, Vol 40, No 4.

[27] Wróbel Z., Koprowski R., 2004, Przetwarzanie obrazu w programie Matlab, EXIT, Warszawa. 